\title{
The association of sleep duration and metabolic syndrome in the Bandare-Kong cohort study, a cross-sectional survey (finding from PERSIAN cohort study)
}

\author{
Shideh Rafati ${ }^{1}$, Maryam Isheh ${ }^{2}$, Abnoos Azarbad ${ }^{3}$, Farhad Ghadiri Soufi ${ }^{4}$, Arash Rahimi ${ }^{4}$ and
} Masoumeh Kheirandish ${ }^{4^{*}}$ (i)

\begin{abstract}
Background: A variety of health problems, such as metabolic syndrome (MetS), have been linked to sleep disorders. While numerous epidemiological studies have shown a U-shaped relationship between sleep duration and poor health outcomes, the results were limited and inconsistent. This study was designed to evaluate the relationship between sleep duration and MetS.

Methods: This population-based study was conducted on the participants aged 35-70 of Bandare-Kong Non-Communicable Diseases (BKNCD) Cohort Study, a part of Prospective Epidemiological Research Studies in IrAN (PERSIAN). MetS was diagnosed according to the National Cholesterol Education Program (NCEP) criteria and the Iranian-specific cut-off for waist circumference ( $\geq 95 \mathrm{~cm}$ ). Sleep information was extracted through a standard questionnaire based on self-reported information. Data were analyzed by $R$ software using generalized additive models (GAMs). A statistically significant level was considered as $\mathrm{P}<0.05$.

Results: A total of 3695 participants were included in the analyses. The mean age was 48.05 years (SD 9.36), and 2067 (55.9\%) were female. The estimated Prevalence of MetS was 35.9\%, and women appeared to be more likely to have MetS than men $(P<0.001)$. There was a non-linear and linear association between sleep duration and the risk of MetS in women and men, respectively. The lowest risk was observed among those with 7-7.5 h of sleep duration per night.

Conclusion: Long sleep duration was associated with increased risk of MetS and higher MetS severity score in both genders, while the short sleep duration increased the risk of Mets as well as MetS severity score just in women. The longitudinal studies would be suggested to assess the relationship between sleep quality and quantity components and MetS.
\end{abstract}

Keywords: Metabolic syndrome, Sleep disorder, Generalized analyzed model, Prospective Epidemiological Research Studies in IrAN (PERSIAN)

*Correspondence: kheirandishm@yahoo.com

${ }^{4}$ Endocrinology and Metabolism Research Center, Hormozgan University of Medical Sciences, Bandar Abbas, Iran

Full list of author information is available at the end of the article

\section{Introduction}

Sleep is an essential lifestyle element that can be surveyed as a significant preventive measure, an indicator to evaluate an individual's current health condition or a health outcome that may lead to other health complications [1]. Therefore, to develop healthy sleep quality, original author(s) and the source, provide a link to the Creative Commons licence, and indicate if changes were made. The images or other third party material in this article are included in the article's Creative Commons licence, unless indicated otherwise in a credit line to the material. If material is not included in the article's Creative Commons licence and your intended use is not permitted by statutory regulation or exceeds the permitted use, you will need to obtain permission directly from the copyright holder. To view a copy of this licence, visit http://creativecommons.org/licenses/by/4.0/. The Creative Commons Public Domain Dedication waiver (http://creativeco mmons.org/publicdomain/zero/1.0/) applies to the data made available in this article, unless otherwise stated in a credit line to the data. 
enhance daytime alertness and overall well-being, sleep-specific guidelines have advised adults to sleep 7 to $8 \mathrm{~h}$ per night. Guidelines also recommend adults to follow a healthy sleep regimen, such as narrowing their daytime naptimes to $30 \mathrm{~min}$, avoiding stimulants like caffeine, especially before their bedtime, avoiding heavy foods like fatty, fried, spicy foods, and also citrus fruits, and finally assuring sufficient exposure to natural sunlight $[2,3]$. Nevertheless, both inadequate and excessive sleep have been regularly reported to be correlated with several health-related conditions like hypertension, obesity, diabetes mellitus [4-6], cardiovascular disorders, stroke, and mortality [5, 7-9].

Metabolic syndrome (MetS) is defined as the group of cardiometabolic risk factors that consists of at least three of the following conditions: high triglyceride levels, raised waist circumference, hypertension, low high-density cholesterol levels, and elevated fasting glucose. Moreover, it is associated with an enhanced risk of cardiovascular disorders and diabetes mellitus [10]. The estimated prevalence of MetS is believed to be approximately one-quarter of the world's population, and therefore it is considered a significant public health challenge [11]. The recent meta-analysis of the Iranian population revealed that at least one-fourth of the population had MetS in Iran [12]. Additionally, the result of Bandare-Kong Non-Communicable Diseases (BKNCD) Cohort Study showed that nearly $34.6 \%$ of the population had MetS [13]. According to the survey data from 2003 to 2012 gathered by the United States National Health and Nutrition Examination, the overall prevalence of metabolic syndrome was $33 \%$. They anticipated that around $35 \%$ of adults and $50 \%$ of individuals aged 60 years or higher had MetS [14]. Based on the data from 9 studies done among European populations, it has been proposed that $38 \%$ of women and $41 \%$ of the men had MetS [15].

Sleep has an essential role in the homeostasis maintenance of the internal environment, balancing physiological, hormonal, and psychological processes [16-18]. Hormonal changes, if not regulated, may lead to a variety of adverse health conditions, such as diabetes, hypertension, cancer, depression, and even mortality [19-23].

While numerous epidemiological studies have shown a U-shaped relationship between sleep duration and lower health outcomes, however, how many hours precisely are regarded harmful and possible gender discrepancies in the association remain ambiguous [24, 25]. Earlier epidemiologic studies have reported that an increase in sleep duration enhanced the risk of MetS [26-28]; however, those results were inconsistent and limited. This study evaluated the association between sleep duration and MetS based on a cross-sectional survey as the first phase of PERSIAN Cohort Study conducted in Bandare-Kong, a city located in the south of Iran.

\section{Methods \\ Study population}

BKNCD provided the data for this descriptive-analytical cross-sectional study. BKNCD $(\mathrm{N}=4063)$ is a large-scale prospective study that took place between November 17, 2016, and November 22, 2018, as part of the Prospective Epidemiological Research Studies in IrAN (PERSIAN) project, which was previously explained in detail [29].

Among subjects, pregnant women and participants using medications causing sleep disorders as well as sedating drugs were excluded. Thus, a total of 3695 subjects were included as the final analytic sample for our study.

\section{Study design}

A face-to-face interview by highly qualified interviewers was conducted using a thorough questionnaire that included demographics, lifestyle variables, eating habits, physical activity, and medical history.

Subjects were weighed on a digital scale (with a measurement accuracy of $0.5 \mathrm{~kg}$ ) while wearing just the bare minimum of clothes and without shoes. The subject's height was measured while standing without shoes and with their shoulders positioned naturally. The waist circumference (WC) of each subject was measured twice, and the average was reported. At the end of numerous consecutive natural breaths, the midway between the top of the iliac crest and the inferior border of the last detectable rib in the midaxillary line at a level parallel to the floor was measured. The maximum circumference of the buttocks was measured at a parallel level to the floor, and the hip circumference (HC) was calculated. To the closest $0.5 \mathrm{~cm}$, all measurements were taken with the same stretch-resistant tape. Subjects stood upright during the measurements, with arms relaxed at the side, feet evenly spread apart, and body weight evenly distributed. The waist-to-hip ratio (WHR) was calculated as WC divided by $\mathrm{HC}$ to the nearest 0.01 .

MetS was defined according to Iranian obesity association guideline when participants met three or more of the following criteria [30]:

1. Abdominal obesity: $W C \geq 95 \mathrm{~cm}$ for men and women

2. Triglycerides $(\mathrm{TG}) \geq 150 \mathrm{mg} / \mathrm{dL}$ or treatment for hypertriglyceridemia.

3. High-density lipoprotein cholesterol (HDLC) $<40 \mathrm{mg} / \mathrm{dL}$ in men and $<50 \mathrm{mg} / \mathrm{dL}$ in women 
4. Systolic blood pressure $(\mathrm{SBP}) \geq 135$, diastolic blood pressure $(\mathrm{DBP}) \geq 85 \mathrm{mmHg}$ or treatment for hypertension

5. Fasting plasma glucose $(\mathrm{FPG}) \geq 100 \mathrm{mg} / \mathrm{dL}$ or treatment for elevated fasting glucose

Blood pressure (BP) was measured in the sitting posture, with feet on the floor and arm at heart level, using a standard mercury sphygmomanometer with a cuff size appropriate for the upper-arm circumference, after $5 \mathrm{~min}$ of rest. The average of two measurement data taken at least 5 min apart was used in the study.

After an overnight fast of 8-h, venous blood samples were taken, and FPG was measured using the glucose oxidase method. After a 12-h overnight fast, venous blood samples were obtained the next day TG, and HDL-C levels were measured using an enzymatic method.

Our study's final model was set according to the following covariates which, all were categorized in the same way as the BKNCD: sex, age, education, and marital status. Education had three categories: less than 6 years, 6-12 years, and more than 12 years. Marital status also had three categories: married, single, and widowed/ divorced.

Further lifestyle covariates were also considered, such as smoking, alcohol consumption status, and physical activity. The International Physical Activity Questionnaire was used for the assessment of physical activity [31]. The weekly average of 24-h physical activities including leisure time activities, work, and exercise was categorized into 3 groups, namely low physical activity (24-36.5 metabolic equivalent of tasks [METs]), moderate (36.6-44.9 METs), and high ( $\geq 45 \mathrm{METs}$ ) [32]. The sleep status information was collected by surveying the following questions: "On average, how many hours do you sleep per day?" Moreover, sedentary behaviors were derived from: "On average, how many hours do you usually lie, sit, or lean per day?" Sleep duration, the time between going to sleep and waking up, was classified as short sleep: sleep $<7 \mathrm{~h}$, normal sleep: sleep between 7 and $9 \mathrm{~h}$, and long sleep: sleep $>9 \mathrm{~h}$.

\section{Data analysis}

The data were examined, and if the sleep duration value was more than $Q 3+3 I Q R$ or less than $Q 1-3 I Q R$, as an outlier was removed ( $Q 1$ : first quartile, Q3: third quartile, IQR: Q3-Q1). To have a better smoothing function in generalized additive models (GAMs), people who sleep between 12 and $13 \mathrm{~h}$ fall into the 12-h sleep group.

Furthermore, outliers were deleted for the five standard components of MetS (waist circumference, SBP, HDL-cholesterol, TG, and fasting plasma glucose), and MetS severity score were calculated using a confirmatory factor analysis approach for the five components of MetS and were treated as a continuous outcome. Moreover, the existence of MetS was considered as a categorical outcome.

Due to the existence possibility of a U-shape association between MetS and sleep duration, as seen in previous studies, the GAMs were used [33].

In the present study, six models of GAM were employed. The MetS (yes/no) was considered a binary response variable for the first three models, whereas the fourth and fifth models MetS severity score as a continuous response variable was used.

Response predictor

Model 1: Metabolic syndrome (yes/no) smooth (sleep).

Model 2: Metabolic syndrome (yes/no) smooth (sleep) for different sexes.

Model 3: Metabolic syndrome (yes/no) age, sex, sitting, smooth (sleep).

Model 4: Metabolic syndrome (yes/no) age, sitting, smooth (sleep *sex).

Model 5: Metabolic syndrome severity score sex, smooth (sleep).

Model 6: Metabolic syndrome severity score age, sex, sitting, smooth (sleep).

Model 7: Metabolic syndrome severity score age, sitting, smooth (sleep "sex).

In model 1, MetS (yes/no) was used as a binary response and a smoothing function of sleep duration as a univariable predictor. Model 2 is the same as Model 1 which has been run in different sexes separately. Model 3 was a multivariable version of model 1; it used binary MetS (yes/no) as a response, but the predictor variables are age, sex, sitting time, smooth (sleep). Model 4, likewise model 3, but the interaction between sex and sleep duration was added. In model 5 , the MetS severity score was used as the continuous response, sex, and smooth (sleep) as predictors. Model 6 is the same as Model 3; just MetS severity score was applied as the continuous response; it used age, sex, sitting time, smooth (sleep) as predictors. The last model was similar to model 6; however, the smoothing function of the interaction between sex and sleep duration was added to the model.

Since there was a high correlation between BMI and WC using Pearson correlation analysis, then BMI was not considered as a variable in the models.

A generalized additive model (GAM) is a generalized linear model in which relationships between the predictors and the dependent variable follow smooth patterns 
that can be linear or non-linear. Age, sex, and sitting time were used to adjust GAMs.

The values of effective degree of freedom (EDF) demonstrate the degree of curvature of the smooth. A value of 1 indicates a linear pattern of relationship. A value of EDF $>1$ shows a more complex relationship between MetS and sleep duration. A $\mathrm{P}<0.05$ was considered as a statistically significant level, and data was analyzed by $R$ software.

\section{Results}

A total of 3695 participants, aged 35-70, were included in the analyses. The mean age was 48.05 years (SD 9.36), and 2067 (55.9\%) were female. Overall, 58.6\% of the participants' education level was below the middle school, and $89.9 \%$ were married. The mean sleep duration was 7.20 (SD 1.46) hours. Furthermore, the estimated Prevalence of MetS was $35.9 \%$, and women appeared to be more likely to have MetS than men $(\mathrm{P}<0.001)$.

More detailed characteristics of the participants, according to metabolic syndrome existence, are presented in Table 1.

Figure 1 illustrates the plots of the estimated smooth function for sleep duration with a $95 \%$ confidence band for model 1 based on GAMs analysis. Figure 1 confirms the non-linear association of sleep duration and the

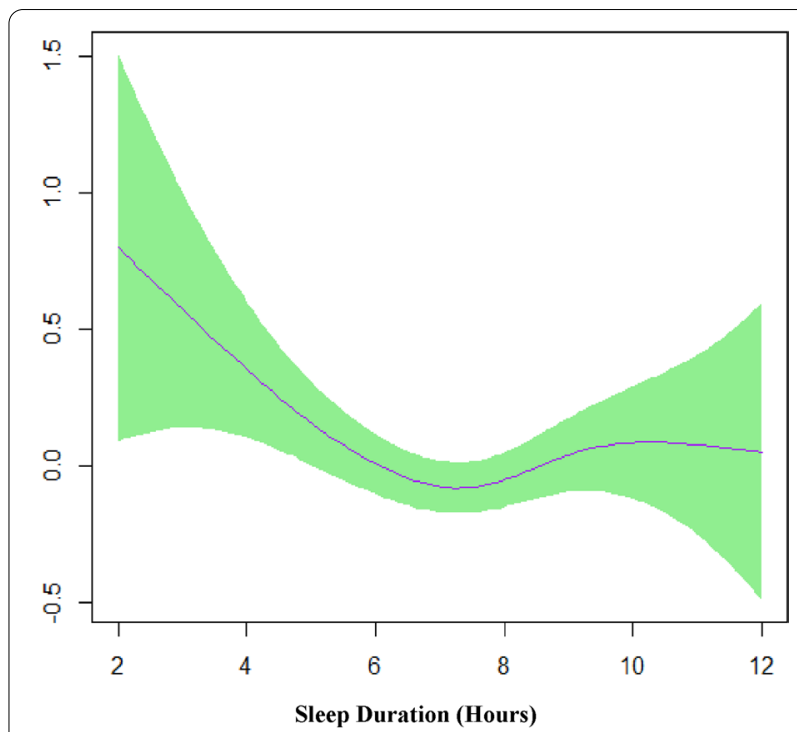

Figure1 Model 1 shows the univariable smooth function of sleep duration with $95 \%$ confidence band ( $E D F=3.09, p=0.02$ )

risk of MetS in the univariate GAMs. The lowest risk is observed among those who had 7-7.5 h of sleep duration per night.

Table 1 Characteristics of participants based on metabolic syndrome

\begin{tabular}{|c|c|c|c|}
\hline \multirow[t]{2}{*}{ Participants' characteristics } & \multicolumn{3}{|c|}{ Metabolic syndrome } \\
\hline & No, $n=2367$ & Yes, $n=1328$ & $\mathbf{p}$ \\
\hline Age, years, mean (SD) & $46.2(8.9)$ & $51.2(9.2)$ & $<0.001$ \\
\hline \multicolumn{4}{|l|}{ Sex, n (\%) } \\
\hline Male & $1224(33.1)$ & $404(10.9)$ & \multirow[t]{2}{*}{$<0.001$} \\
\hline Female & $1143(30.9)$ & $924(25.1)$ & \\
\hline \multicolumn{4}{|l|}{ Marital Status, n (\%) } \\
\hline Single & $63(1.7)$ & $25(0.7)$ & \multirow[t]{3}{*}{$<0.001$} \\
\hline Married & $2156(58.3)$ & $1167(31.6)$ & \\
\hline Widowed/divorced & $148(4.0)$ & $136(3.7)$ & \\
\hline \multicolumn{4}{|l|}{ Education, $n$ (\%) (years) } \\
\hline$<6$ & $1244(33.7)$ & $923(24.9)$ & \multirow[t]{3}{*}{$<0.001$} \\
\hline $6-12$ & $882(23.9)$ & $328(8.9)$ & \\
\hline$>12$ & $241(6.5)$ & $77(2.1)$ & \\
\hline Sitting, hours/day, mean (SD) & $9.5(2.5)$ & $10.5(2.6)$ & $<0.001$ \\
\hline Sleep duration, hours, mean (SD) & $7.2(1.4)$ & $7.1(1.5)$ & 0.2 \\
\hline Diastolic blood pressure, mmHg, mean (SD) & $74.6(9.5)$ & $81.1(10.6)$ & $<0.001$ \\
\hline Systolic blood pressure, mmHg, mean (SD) & $113.9(14.5)$ & $127.0(18.9)$ & $<0.001$ \\
\hline Fasting blood glucose, mg/dL, mean (SD) & $96.5(27.7)$ & $128.2(55.7)$ & $<0.001$ \\
\hline High density lipoprotein, mg/dL, mean (SD) & $49.4(10.4)$ & $45.1(10.8)$ & $<0.001$ \\
\hline Triglyceride, mg/dL, mean (SD) & $113.8(60.7)$ & $177.0(112.2)$ & $<0.001$ \\
\hline Waist circumference, cm, mean (SD) & $90.1(11.2)$ & $99.9(10.2)$ & $<0.001$ \\
\hline
\end{tabular}

SD: standard deviation 
Figure 2 shows the plots of the predicted smooth relationship between MetS (yes/no) and sleep duration separately for males and females in model 2 . This figure confirms a non-linear association of sleep duration and MetS in females and a linear pattern in males.

Table 2 indicates the relationship between MetS and independent variables in model 3 by GAMs output. Based on Table 2, an EDF of 1.49 indicates a semi-linear fit between sleep duration and MetS. Model 4 represented two different smoothing shapes; an almost U-shaped in women and a linear one in men (Fig. 3).

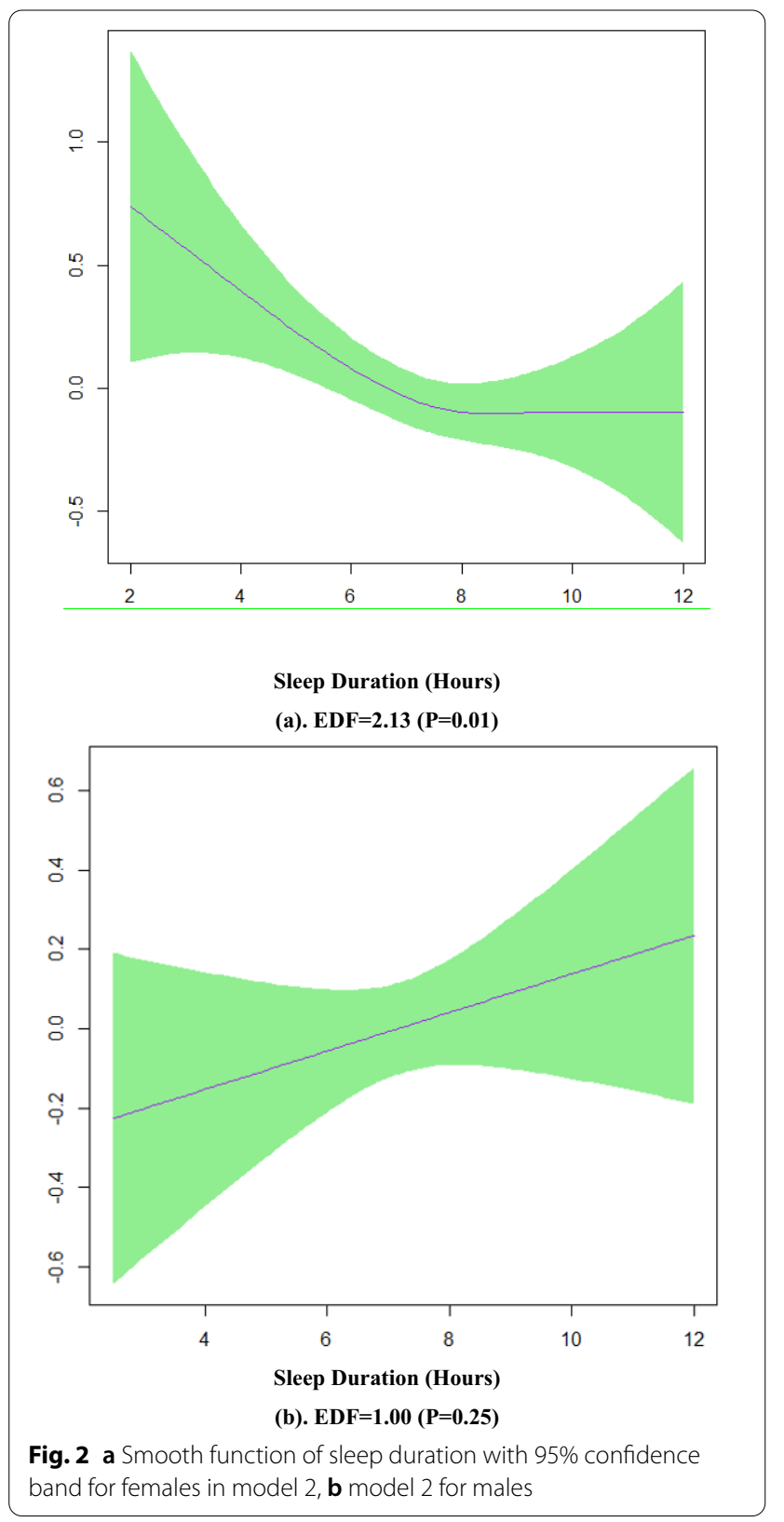

Table 2 Association of metabolic syndrome and independent variables measured by multivariable generalized additive model 3

\begin{tabular}{llr}
\hline $\mathbf{R}^{2}=\mathbf{0 . 1 2 1}$ & Outcome: metabolic syndrome \\
\cline { 2 - 3 } $\mathbf{n}=\mathbf{3 6 9 5}$ & $\boldsymbol{\beta}$ & $\mathbf{P}$ \\
\hline Age & 0.06 & $<0.001$ \\
Sex & & \\
Male & Reference group & $<0.001$ \\
Female & 0.91 & $<0.001$ \\
Sitting & 0.08 & 0.114 \\
Sleep & Smooth curve, EDF $=1.49$ & \\
\hline
\end{tabular}

Figure 4 presents the plots of the predicted smooth relationship between MetS severity score and sleep duration separately for males and females in model 5 . This

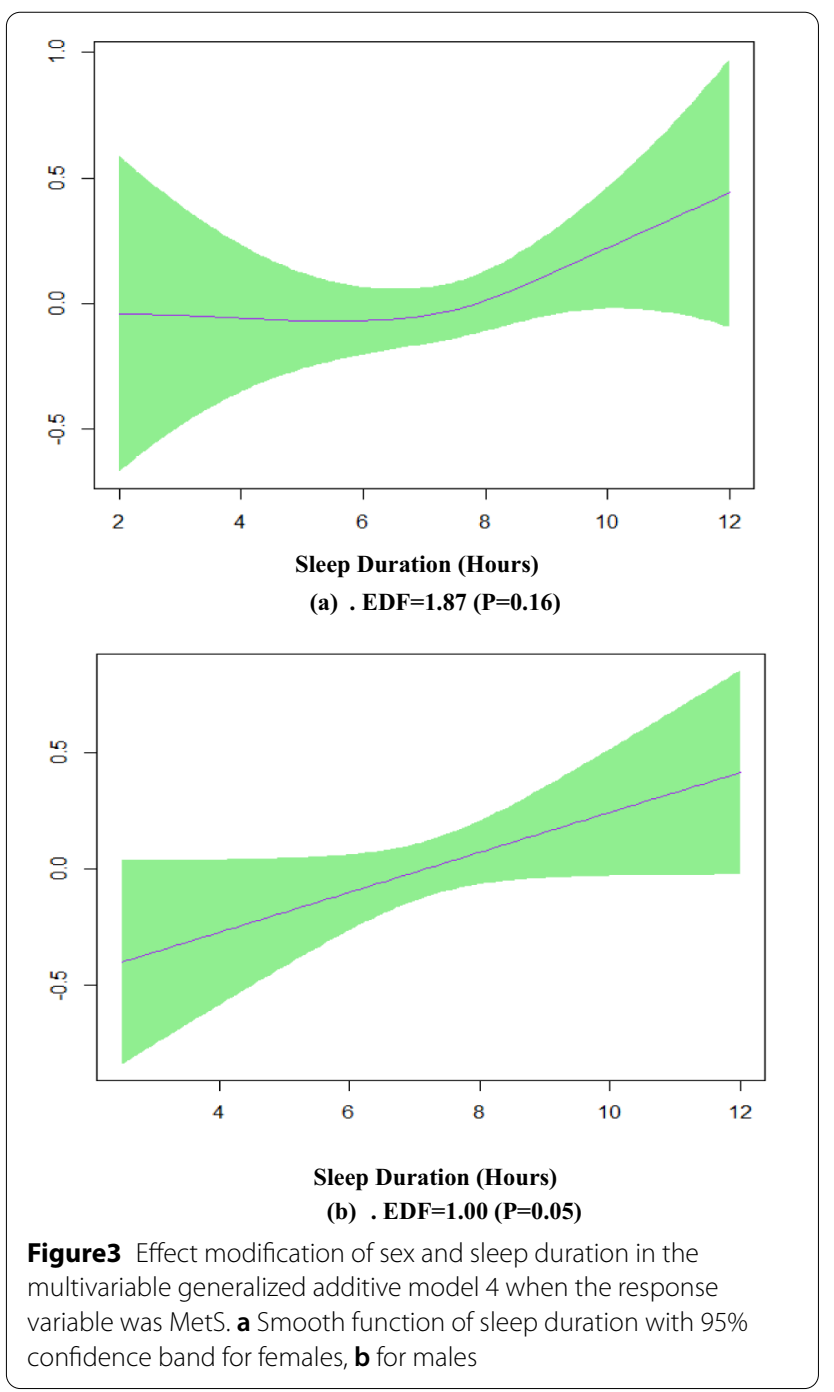




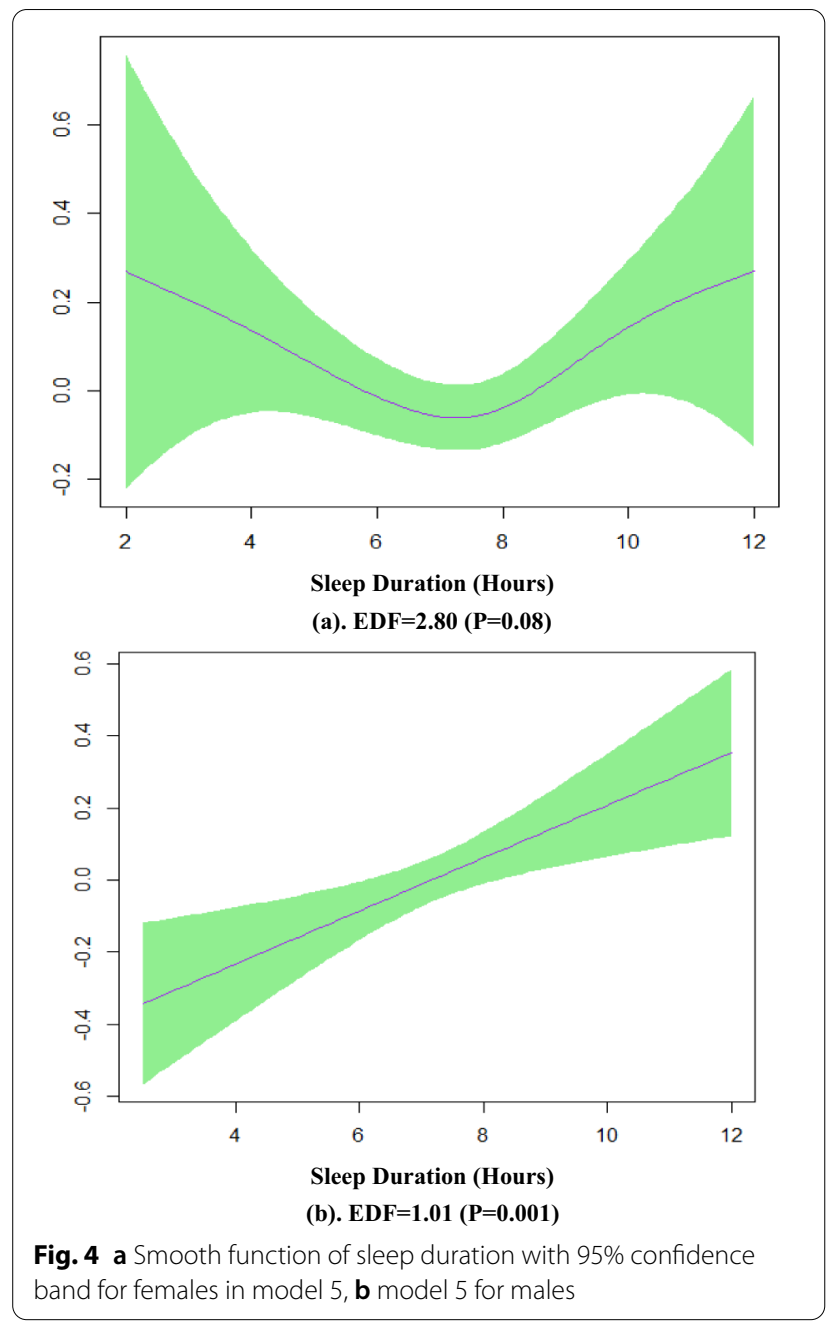

figure confirms a U-shape association of sleep duration and MetS severity score in females and a linear pattern in males.

Table 3 shows the output of model 6 , a significant EDF of 1.85 reveals a non-linear pattern between sleep duration and MetS severity score. A significant EDF of 1.85

Table 3 Association of metabolic syndrome and independent variables measured by model 6

\begin{tabular}{lll}
\hline $\mathbf{R}^{\mathbf{2}}=\mathbf{0 . 0 6}$ & \multicolumn{2}{l}{ Outcome: metabolic syndrome severity score } \\
\cline { 2 - 3 } $\mathbf{n}=\mathbf{3 6 9 5}$ & $\boldsymbol{\beta}$ & $\mathbf{P}$ \\
\hline Age & 0.02 & $<0.001$ \\
Sex & & \\
$\quad$ Male & Reference group & \\
Female & 0.37 & $<0.001$ \\
Sitting & 0.06 & $<0.001$ \\
Sleep & Smooth curve, EDF $=1.85$ & $<0.001$ \\
\hline
\end{tabular}

indicates an almost non-linear fit between sleep duration and MetS severity score.

Furthermore, the multivariate GAM model 7 shows two different smoothing patterns for metabolic syndrome severity score; a non-linear fit in women $(E D F=2.70$, $\mathrm{p}<0.001)$ and a linear pattern in men $(E D F=1.02$, $\mathrm{p}<0.001$ ) (Fig. 5).

\section{Discussion}

To the best of our knowledge, this is the first survey of the BKNCD Cohort Study database that examined the association between sleep duration and risk of MetS among males and females separately in the south of Iran. Our research presented the most up-to-date estimates of

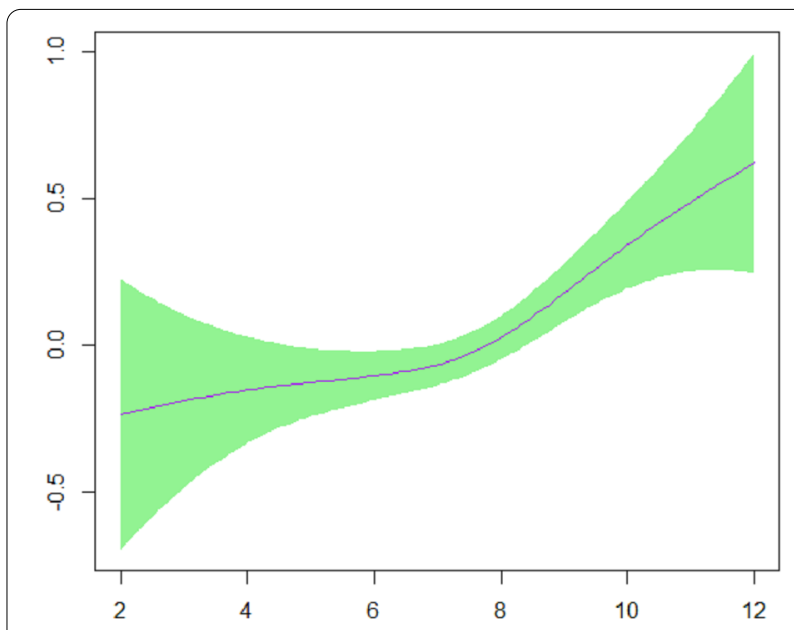

(a)

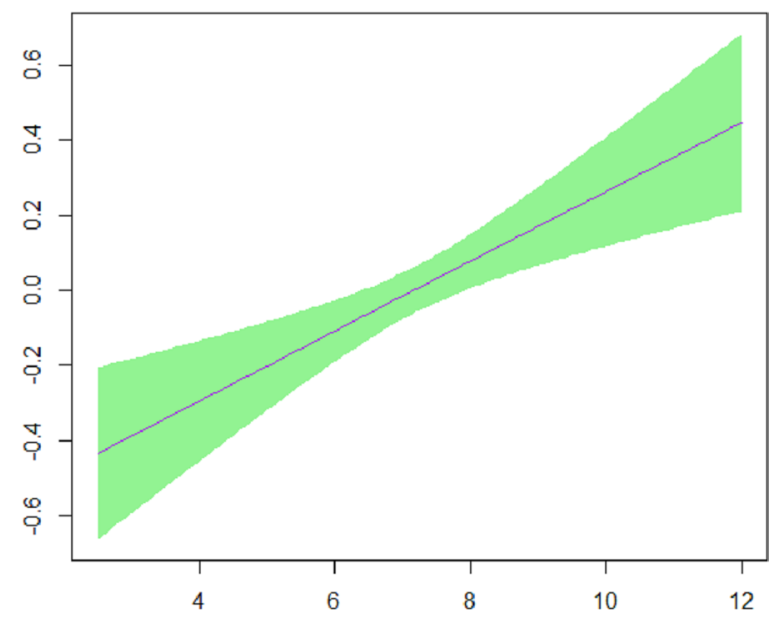

(b)

Fig. 5 Effect modification of sex and sleep duration in the multivariate generalized additive model 7 when the response variable was metabolic syndrome severity score. a Smooth function of sleep duration with $95 \%$ confidence band for females, $\mathbf{b}$ for males 
MetS prevalence among people aged 35 to 70 . MetS was projected to affect $35.9 \%$ of the population, with women being more affected than men. The current cross-sectional analysis found statistically significant associations between short and long sleep periods and MetS risk, supporting the existence of a U-shape relationship in women and a linear association in men.

According to the univariate model, people who slept $7-7.5 \mathrm{~h}$ a night have the lowest chance of MetS. Similarly, those who slept less than or more than $7.5 \mathrm{~h}$ had a higher risk than those who slept for $7 \mathrm{~h}$.

The current study showed that long sleep was associated with MetS in both genders. In our research, the gender interaction in the relationship between sleep duration and MetS was statistically significant; however, a similar study finding displayed that long sleep was associated with MetS in women only [34].

Several mechanisms for MetS associated sleep disorders have been proposed, including endocrinologic, immunologic, biologic, hormonal, and metabolic processes. Sleeping for less than $7 \mathrm{~h}$ can provoke mutual changes in circulating leptin and ghrelin levels, resulting in high ghrelin and low leptin levels [35], increasing appetite, caloric intake, and lowering energy expenditure [30,36], facilitating an increase in central obesity, one of the main components of MetS. Sleep deprivation has also been associated with decreased glucose tolerance, associated with an increased risk of hypertension and diabetes [37]. Increased cortisol levels, which can raise fasting glucose levels, are another endocrinologic consequence of sleep restriction [38]. In addition, clinical studies have shown that sleep deprivation increases levels of Interleukin 6 (IL-6) and C-reactive protein (CRP), both of which have been linked to MetS constituents through increased insulin resistance [39].

Circadian regulation of energy metabolism and hormonal secretion are the possible causal mechanism [16, 17, 39]. Sleep disruption has been linked to impaired metabolism and appears to play a role in the pathogenesis of metabolic diseases. Circadian rhythmicity and autonomic balance can be disrupted due to sleep timing and duration changes, resulting in diurnal cardiac output rhythm disturbance and increased blood pressure variability [40, 41].

Among other potential mechanisms of sleep-MetS interaction, it is worth noting that stage 3 of sleep is the most important since this is when the growth hormone $(\mathrm{GH})$ and $\mathrm{GH}$ releasing hormone (GHRH) are released. They provoke fat loss, bone growth, and general repair and regeneration. Before midnight, the longest part of stage 3 sleep occurs. Sleep deprivation will inhibit the most potent GH pulse, raising the risk of MetS [33].
Only a few studies have documented gender-stratified sleep associations with MetS is one of the current study's key strengths. A meta-analysis consisting of 12 crosssectional and three cohort studies from North America, Europe, and Asia found that sleep duration of fewer than $5 \mathrm{~h}$ and greater than $8 \mathrm{~h}$ were both linked to MetS but with no gender differences [28].

Furthermore, we were able to support the idea that both short and long sleep are linked to MetS in women in our research, but a recent meta-analysis, while finding a dose-response association between short and long sleep and MetS, was unable to support the idea that long sleep is linked to MetS as well [42]. Our findings indicated a linear association between sleep duration and metabolic syndrome in men. On the other hand, long sleep duration increased the risk of metabolic syndrome and metabolic syndrome severity score while short sleep duration did not affect. A Korean study found that a sleep duration greater than or equal to $9 \mathrm{~h}$ was linked to MetS, but no connection was found between short sleep and MetS [43].

Among the studies' other strengths, we can point to the analysis process and the fact that the MetS severity score was used. Using the MetS severity score improved the association's strength because it provided a continuous measure of the risk of metabolic status, whereas MetS definition using qualitative criteria is not an accurate measurement compare with MetS score. Furthermore, all five elements of the MetS, whether high, borderline, or low, contribute to the MetS severity score measurement. In contrast, only the high components identified by the threshold specified in the definition are considered to diagnose MetS [44]. This limitation could be resolved by measuring the MetS severity score, including the actual measurements of all five components. Other studies have shown the accuracy of the MetS severity score in estimating the likelihood of health outcomes [45, 46]. Furthermore, the generalized additive models' application to investigate the relationship between sleep and MetS and MetS severity score improved the risk adjustment compared to other models $[47,48]$.

While our research shows a connection between sleep duration and MetS, it also has several limitations. First, as the current study is a cross-sectional analysis, it restricts us from supposing the causative link between sleep duration and MetS. Hence, this study should be conducted jointly into a prospective cohort study.

Second, rather than using objective measurements like an actigraph or polysomnography, sleep duration was measured using self-report questionnaires. As a result, knowledge bias may occur based on how much sleep one believes they got [49]. 
Third, the total amount of sleep time measured may include both nighttime and naptime sleep. Since daytime napping has previously been linked to lower sleep quality, shorter sleep length, and, as a result, cardiovascular risk factors [50], it would be beneficial to differentiate between naptime and nighttime sleep to determine their effect on health separately.

Fourth, data on sleep quality, sleep disruptions, insomnia symptoms as well as sleep apnea were not analyzed because they were not available. Sleep disruptions have been linked to metabolic disorders in previous studies [51], highlighting the significance of considering sleep quality when assessing the impact of sleep on overall health.

Eventually, longitudinal studies in different populations could help to improve the reliability and generalizability of this study's findings.

\section{Conclusion}

Findings represented a linear pattern between the risk of MetS as well as higher MetS severity score and sleep duration in men and an almost u-shaped pattern in women. Of note of the growing prevalence of metabolic syndrome and its role as a significant risk factor of cardiometabolic disease, the sleep disorder should be considered a health priority to reduce and improve metabolic syndrome. It would be suggested to conduct longitudinal studies to evaluate the relationship between sleep quality components, including sleep duration and MetS in the next phases of the study.

\begin{abstract}
Abbreviations
PERSIAN: Prospective Epidemiological Research Studies in IrAN; BKNCD: Bandare-Kong Non-Communicable Diseases; ADA: American Diabetes Association; MetS: Metabolic syndrome; ATP III: Adult Treatment Panel III; BMI: Body mass index; CVD: Cardiovascular disease; BP: Blood pressure; DBP: Diastolic blood pressure; SBP: Systolic blood pressure; FPG: Fasting plasma glucose; HC: Hip circumference; TC: Total cholesterol; WC: Waist circumference; WHO: World Health Organization; WHR: Waist-to-hip ratio; TG: Triglyceride; HDL: Highdensity lipoprotein; LDL: Low-density lipoprotein; METs: Metabolic equivalent of task; GAMs: Generalized additive models; OR: Odds ratio; Cl: Confidence interval; GH: Growth hormone; GHRH: Growth hormone-releasing hormone; CRP: Creactive protein.
\end{abstract}

\section{Acknowledgements}

BKNCD is a part of PERSIAN national cohort and we would like to appreciate Professor Reza Malekzadeh, Deputy of Research and Technology at the Ministry of Health and Medical Education of Iran and Director of the PERSIAN cohort, and also Dr. Hossein Poustchi, Executive Director of the PERSIAN cohort and Azim Nejatizadeh Director of BKNCD for all their supports during design and running of BKNCD.

\section{Authors' contributions}

MK designed and supervised the study. SR performed the statistical analysis and wrote the manuscript. MI and AA wrote the manuscript. FGS was consulted on the possible associated factors to be taken into account. AR revised the manuscript. All authors read and approved the final manuscript.

\section{Funding}

The Iranian Ministry of Health and Medical Education has funded the study in the PERSIAN Cohort through Grant Number 700/534.

\section{Availability of data and materials}

The datasets used and/or analyzed during the current study are available from the corresponding author on reasonable request.

\section{Declarations}

\section{Ethics approval and consent to participate}

The study was approved by the Institutional Review Board of Hormozgan University of Medical Sciences (IR.HUMS.REC.1399.305), and it complies with the statements of the Declaration of Helsinki. Informed consent was obtained from all subjects and from a parent and/or legal guardian for vulnerable population.

\section{Competing interests}

The authors declare that they have no competing interests.

\section{Author details}

${ }^{1}$ Social Determinants in Health Promotion Research Center, Hormozgan University of Medical Sciences, Bandar Abbas, Iran. ${ }^{2}$ Student Research Committee, Faculty of Medicine, Hormozgan University of Medical Sciences, Bandar Abbas, Iran. ${ }^{3}$ Department of Pharmacology, Faculty of Pharmacy, Eastern Mediterranean University, via Mersin 10, Famagusta, North Cyprus, Turkey. ${ }^{4}$ Endocrinology and Metabolism Research Center, Hormozgan University of Medical Sciences, Bandar Abbas, Iran.

Received: 7 August 2021 Accepted: 12 October 2021

Published online: 20 October 2021

\section{References}

1. Health, U.S.D.o., S. Human. Office of disease prevention and health promotion. Healthy people, 2020. 2010.

2. Hirshkowitz M, et al. National Sleep Foundation's sleep time duration recommendations: methodology and results summary. Sleep Health. 2015;1(1):40-3.

3. Ohayon M, et al. National Sleep Foundation's sleep quality recommendations: first report. Sleep Health. 2017;3(1):6-19.

4. Kim K, et al. Association between sleep duration, fat mass, lean mass and obesity in Korean adults: the fourth and fifth Korea National Health and Nutrition Examination Surveys. J Sleep Res. 2017;26(4):453-60.

5. Magee CA, et al. Short and long sleep duration are associated with prevalent cardiovascular disease in Australian adults. J Sleep Res. 2012;21(4):441-7.

6. Itani $\mathrm{O}$, et al. Short sleep duration and health outcomes: a systematic review, meta-analysis, and meta-regression. Sleep Med. 2017;32:246-56.

7. Shen X, Wu Y, Zhang D. Nighttime sleep duration, 24-hour sleep duration and risk of all-cause mortality among adults: a meta-analysis of prospective cohort studies. Sci Rep. 2016;6(1):1-8.

8. Liu T-Z, et al. Sleep duration and risk of all-cause mortality: a flexible, nonlinear, meta-regression of 40 prospective cohort studies. Sleep Med Rev. 2017;32:28-36.

9. Gallicchio L, Kalesan B. Sleep duration and mortality: a systematic review and meta-analysis. J Sleep Res. 2009;18(2):148-58.

10. Grundy SM. Metabolic syndrome scientific statement by the american heart association and the national heart, lung, and blood institute. Arterioscler Thromb Vasc Biol. 2005;25(11):2243-4.

11. Saklayen MG. The global epidemic of the metabolic syndrome. Curr Hypertens Rep. 2018;20(2):1-8.

12 Fatahi A, Doosti-Irani A, Cheraghi Z. Prevalence and incidence of metabolic syndrome in Iran: a systematic review and meta-analysis. Int J Prev Med. 2020;11:64.

13. Zoghi G, Kheirandish M. Prevalence of type 2 diabetes, obesity, central obesity, and metabolic syndrome in a South Coastal Region, Iran, the PERSIAN Bandare Kong cohort study: a brief report. Hormozgan Med J. 2021;25(1):42-4. 
14. Aguilar $M$, et al. Prevalence of the metabolic syndrome in the United States, 2003-2012. JAMA. 2015;313(19):1973-4.

15. Gao W. Does the constellation of risk factors with and without abdominal adiposity associate with different cardiovascular mortality risk? Int J Obes. 2008;32(5):757-62.

16. Potter GDM, et al. Circadian rhythm and sleep disruption: causes, metabolic consequences, and countermeasures. Endocr Rev. 2016;37(6):584-608.

17. Morris CJ, Aeschbach D, Scheer FAJL. Circadian system, sleep and endocrinology. Mol Cell Endocrinol. 2012;349(1):91-104.

18. Selvi Y, et al. The effects of individual biological rhythm differences on sleep quality, daytime sleepiness, and dissociative experiences. Psychiatry Res. 2017;256:243-8.

19. Guo X, et al. Epidemiological evidence for the link between sleep duration and high blood pressure: a systematic review and meta-analysis. Sleep Med. 2013;14(4):324-32.

20. Wang J, et al. Sleep duration and risk of diabetes: observational and Mendelian randomization studies. Prev Med. 2019;119:24-30.

21. Shen J, et al. Sleep duration and risk of cancer in the Mexican American Mano-a-Mano Cohort. Sleep Health. 2019;5(1):78-83.

22. Ouyang P, Sun W. Depression and sleep duration: findings from middleaged and elderly people in China. Public Health. 2019;166:148-54.

23. Cai $\mathrm{H}$, et al. Sleep duration and mortality: a prospective study of 113,138 middle-aged and elderly Chinese men and women. Sleep. 2015;38(4):529-36.

24 da Silva AA, et al. Sleep duration and mortality in the elderly: a systematic review with meta-analysis. BMJ Open. 2016;6(2):e008119.

25. Cappuccio FP, et al. Sleep duration and all-cause mortality: a systematic review and meta-analysis of prospective studies. Sleep. 2010;33(5):585-92.

26. Jike $M$, et al. Long sleep duration and health outcomes: a systematic review, meta-analysis and meta-regression. Sleep Med Rev. 2018:39:25-36

27. Xi B, et al. Short sleep duration predicts risk of metabolic syndrome: a systematic review and meta-analysis. Sleep Med Rev. 2014;18(4):293-7.

28. Ju SY, Choi WS. Sleep duration and metabolic syndrome in adult populations: a meta-analysis of observational studies. Nutr Diabetes. 2013;3(5):e65-e65.

29. Poustchi $\mathrm{H}$, et al. Prospective epidemiological research studies in Iran (the PERSIAN cohort study): rationale, objectives, and design. Am J Epidemiol. 2018;187(4):647-55.

30. Azizi F, et al. Appropriate definition of metabolic syndrome among Iranian adults: report of the Iranian National Committee of Obesity. 2010.

31. Hagströmer M, Oja P, Sjöström M. The International Physical Activity Questionnaire (IPAQ): a study of concurrent and construct validity. Public Health Nutr. 2006:9(6):755-62.

32. Pasdar Y, et al. Cohort profile: Ravansar non-communicable disease cohort study: the first cohort study in a Kurdish population. Int J Epidemiol. 2019:48(3):682-683f.

33. Smiley A, King D, Bidulescu A. The association between sleep duration and metabolic syndrome: the NHANES 2013/2014. Nutrients. 2019;11(11):2582
34. Yoon $\mathrm{H}-\mathrm{S}$, et al. Associations of sleep duration with metabolic syndrome and its components in adult Koreans: from the health examinees study. Sleep Biol Rhythms. 2016;14(4):361-8.

35. Spiegel K, et al. Brief communication: sleep curtailment in healthy young men is associated with decreased leptin levels, elevated ghrelin levels, and increased hunger and appetite. Ann Intern Med. 2004; 141(11):846-50.

36. Zimberg IZ, et al. Short sleep duration and obesity: mechanisms and future perspectives. Cell Biochem Funct. 2012;30(6):524-9.

37. Yoda K, et al. Association between poor glycemic control, impaired sleep quality, and increased arterial thickening in type 2 diabetic patients. PLoS ONE. 2015;10(4):e0122521.

38. Spiegel K, Leproult R, Van Cauter E. Impact of sleep debt on metabolic and endocrine function. The lancet. 1999:354(9188):1435-9.

39. Poggiogalle $E$, Jamshed $H$, Peterson CM. Circadian regulation of glucose, lipid, and energy metabolism in humans. Metabolism. 2018:84:11-27.

40. Gangwisch JE. A review of evidence for the link between sleep duration and hypertension. Am J Hypertens. 2014;27(10):1235-42.

41. Wang Y, et al. Relationship between duration of sleep and hypertension in adults: a meta-analysis. J Clin Sleep Med. 2015:11(9):1047-56.

42 Iftikhar $\mathrm{H}$, et al. Sleep duration and metabolic syndrome. An updated dose-risk metaanalysis. Ann Am Thorac Soc. 2015;12(9):1364-72.

43. Stefani KM, et al. The influence of sex and age on the relationship between sleep duration and metabolic syndrome in Korean adults. Diabetes Res Clin Pract. 2013:102(3):250-9.

44. Gurka MJ, et al. Independent associations between a metabolic syndrome severity score and future diabetes by sex and race: the atherosclerosis risk in communities study and Jackson heart study. Diabetologia. 2017;60(7):1261-70.

45. Dimitrov BD, et al. Metabolic syndrome severity score: range and associations with cardiovascular risk factors. Arch Med Sci Atheroscler Dis. 2016;1(1):e90

46. Guo Y, et al. Assessing the added predictive ability of a metabolic syndrome severity score in predicting incident cardiovascular disease and type 2 diabetes: the atherosclerosis risk in communities study and Jackson heart study. Diabetol Metab Syndr. 2018;10(1):1-14.

47. Benedetti A, Abrahamowicz M. Using generalized additive models to reduce residual confounding. Stat Med. 2004;23(24):3781-801.

48. Moore $L$, et al. A comparison of generalized additive models to other common modeling strategies for continuous covariates: implications for risk adjustment. J Biomet Biostat. 2011;2(109):2.

49. Bin YS. Short sleep duration as a health risk factor: what is new? Sleep Med. 2017;100(32):257-8.

50. Owens JF, et al. Napping, nighttime sleep, and cardiovascular risk factors in mid-life adults. J Clin Sleep Med. 2010;6(4):330-5.

51. Grandner MA, et al. Sleep disturbance is associated with cardiovascular and metabolic disorders. J Sleep Res. 2012:21(4):427-33.

\section{Publisher's Note}

Springer Nature remains neutral with regard to jurisdictional claims in published maps and institutional affiliations.

\footnotetext{
Ready to submit your research? Choose BMC and benefit from:

- fast, convenient online submission

- thorough peer review by experienced researchers in your field

- rapid publication on acceptance

- support for research data, including large and complex data types

- gold Open Access which fosters wider collaboration and increased citations

- maximum visibility for your research: over 100M website views per year
}

At BMC, research is always in progress.

Learn more biomedcentral.com/submissions 